\title{
Segmentation of Biological Target Volumes on Multi-tracer PET Images Based on Information Fusion for Achieving Dose Painting in Radiotherapy
}

\author{
Benoît Lelandais $^{1}$, Isabelle Gardin ${ }^{1,2}$, Laurent Mouchard ${ }^{1}$, \\ Pierre Vera ${ }^{1,2}$, and Su Ruan ${ }^{1}$ \\ 1 University of Rouen, LITIS EA4108, QuantIF, \\ 22 bd Gambetta, 76183 Rouen Cedex, France \\ \{benoit.lelandais, laurent.mouchard, su.ruan\}@univ-rouen.fr \\ http://www.litislab.eu/front-page/themes/quantif \\ ${ }^{2}$ Centre Henri-Becquerel, Dept of Nuclear Medicine \\ 1 rue d'Amiens, 76038 Rouen Cedex 1, France \\ $\{$ isabelle.gardin, pierre.vera\}@chb.unicancer.fr
}

\begin{abstract}
Medical imaging plays an important role in radiotherapy. Dose painting consists in the application of a nonuniform dose prescription on a tumoral region, and is based on an efficient segmentation of Biological Target Volumes (BTV). It is derived from PET images, that highlight tumoral regions of enhanced glucose metabolism (FDG), cell proliferation (FLT) and hypoxia (FMiso). In this paper, a framework based on Belief Function Theory is proposed for BTV segmentation and for creating 3D parametric images for dose painting. We propose to take advantage of neighboring voxels for BTV segmentation, and also multitracer PET images using information fusion to create parametric images. The performances of BTV segmentation was evaluated on an anthropomorphic phantom and compared with two other methods. Quantitative results show the good performances of our method. It has been applied to data of five patients suffering from lung cancer. Parametric images show promising results by highlighting areas where a high frequency or dose escalation could be planned.
\end{abstract}

Keywords: Dose Painting, Positron Emission Tomography, Information Fusion, Segmentation, Belief Function Theory.

\section{Introduction}

Medical imaging plays an important role in radiotherapy for treatment planning with the delineation of Gross Tumor Volume on Computed Tomography (CT) images. Ling et al. [1] have proposed the concept of dose painting, consisting of the application of a nonuniform dose prescription based on the biological characteristics of the tumor. Positron Emission Tomography (PET) is used for sub-volumes definition, also called Biological Target Volumes (BTV) 
corresponding, for instance, to tumoral regions. Different radiotracers are used for visualising specific properties: ${ }^{18} \mathrm{~F}$-FluoroDesoxyGlucose (FDG) for enhanced glucose metabolism, ${ }^{18} \mathrm{~F}$-FLuoroThymidine (FLT) for cell proliferation and ${ }^{18} \mathrm{~F}$ FluoroMisonidazol (FMiso) for hypoxic regions (lack of oxygen). An increase of the frequency of dose delivery on high proliferative cells could be planned, as well as a dose escalation on hypoxic regions regarding their radioresistance.

If the concept of dose painting is promising, it raises two methodological problems. First, it might be difficult to correctly segment BTVs because of the poor quality of PET images (significant noise), Partial Volume Effect (PVE) and possibly, a low contrast between normal and pathological tissues. Second, the large number of images that a radiation oncologist has at his disposal makes complex the decision-making when considering nonuniform dose prescription.

Although several algorithms have been proposed in the literature to delineate FDG-PET positive tissues, there is no consensus on this issue. One can quote thresholding methods [2], watershed algorithms [3], or methods based on probability measures [4] or membership degree estimation [5]. On the other side, few authors have proposed a method for the segmentation of FLT and FMiso-PET positive tissues. Even if, in first approximation, the level of noise, PVE and contrast between normal and pathological tissues can be considered in a same order of magnitude in FDG and FLT-PET images, performances of algorithms have to be evaluated on images of both tracers. Moreover, FMiso-PET images generally have a low contrast and an important noise. Here again, a specific validation is mandatory.

Our goal is to propose a framework based on Belief Function Theory (BFT) for the segmentation of BTV and creation of 3D parametric images to help the radiation oncologist for dose painting, in a context of noise, PVE, and muti-tracer images. In BFT, noise and PVE lead to partial knowledges and are considered as imperfect information. This theory was chosen for its ability to manage partial knowledge and to merge information coming from several sources [6 9]. To this end, we propose to benefit from neighborhood information in each image for BTV segmentation, and also to fuse multi-tracer PET images for 3D parametric images creation for dose painting.

\section{Method}

\subsection{Belief Function Theory (BFT)}

Frame of Discernment. Let $\Omega=\left\{\omega_{1}, \omega_{2}, \ldots, \omega_{C}\right\}$ be a finite set of classes, called the frame of discernment. Partial knowledge flowing out from information sources are taken into account using BFT by assigning masses, $m$, also called Basic Belief Assignments (BBAs), over different subsets of $\Omega . m$ is defined as a mapping of hypotheses from $2^{\Omega}=\left\{\varnothing,\left\{\omega_{1}\right\},\left\{\omega_{2}\right\}, \ldots,\left\{\omega_{C}\right\},\left\{\omega_{1}, \omega_{2}\right\}, \ldots, \Omega\right\}$ to $[0,1]$ verifying $\sum_{A \subseteq \Omega} m(A)=1$. The mass $m(\Omega)$ represents the degree of ignorance $(m(\Omega)=1$ : total ignorance) and the mass $m(\varnothing)$ represents the conflict between sources. 
In the hypotheses, one can distinguish singletons, such as $\left\{\omega_{1}\right\}$, from disjunctions, such as $\left\{\omega_{1}, \omega_{2}\right\}$ corresponding to a set of exclusive classes. Singletons allow to represent uncertainty as in probability theory. A BBA is considered as uncertain when masses are approximatively equals over singletons, corresponding to equiprobability in probability theory. Disjunctions allow to represent imprecision. A BBA is considered as imprecise when non-zero masses are assigned over disjunctions, meaning that we do not take part in favor of one class or an other.

Discounting. Having knowledge about the reliability of a source, the discounting process allows to reduce its reliability [7]. It consists in transferring a part of belief either on $X=\varnothing$ or $X=\Omega$ according to a coefficient $\alpha$ in $[0,1]$, such that:

$$
\begin{aligned}
m^{\prime}(A) & =\alpha \cdot m(A), \quad \forall A \neq X, \\
m^{\prime}(X) & =1-\alpha(1-m(X)),
\end{aligned}
$$

Information Fusion. When at least two sources contributing to the same BBA estimation are available, their fusion can be achieve using combination rules.

Let $S_{1}$ and $S_{2}$ be two information sources such that, at least, one is reliable. The disjunctive rule is given by:

$$
m_{S_{1} \oplus S_{2}}(A)=\sum_{B \cup C=A} m_{1}(B) \cdot m_{2}(C) .
$$

It makes imprecision growing when sources are discordant, transferring masses over disjunctions.

Let $S_{1}$ and $S_{2}$ be two reliable sources of information. The conjunctive combination rule is given by:

$$
m_{S_{1} \oplus S_{2}}(A)=\sum_{B \cap C=A} m_{1}(B) \cdot m_{2}(C) .
$$

It allows to reduce uncertainty and imprecision by transferring belief masses on intersections between hypotheses. When the information contained in sources are conflicting, non-zero masses are transfered on $\varnothing$. In order to satisfy $m(\varnothing)=0$, the Dempster's rule is used [6] and consists in normalizing the conjunctive rule by $\kappa=m_{S_{1} \odot S_{2}}(\varnothing)$ :

$$
m_{S_{1} \oplus S_{2}}(A)=\frac{1}{1-\kappa} \sum_{B \cap C=A} m_{1}(B) \cdot m_{2}(C) .
$$

\subsection{Segmentation of Biological Target Volumes (BTV)}

Frame of Discernment and Information Sources. We propose to represent mono-tracer PET images (FDG, FLT or FMiso) by two classes: one corresponds to low uptake $(l u)$ region and the other, to high uptake $(h u)$ region. Thus, the frame of discernment is represented by $\Omega=\{l u, h u\}$ and each voxel $V_{i}$ is a source of information which is associated to a specific BBA represented by four masses: $m_{V_{i}}(\varnothing), m_{V_{i}}(\{l u\}), m_{V_{i}}(\{h u\})$ and $m_{V_{i}}(\{l u, h u\})$. 
BBA Estimation. This estimation can be separated in two steps leading each time to a set of BBAs.

At the first step, the BBAs of voxels according to $\{l u\},\{h u\}$, and $\{l u, h u\}$ hypotheses are determined using a modified Fuzzy C-Means (FCM) algorithm [10]. The modification consists in integrating a disjunctive combination of neighboring voxels (Eq. 2) inside the iterative process. This combination tends firstly to transfer the masses over the disjunction $\{l u, h u\}$ for imperfect data due to the ambiguity of the information in the voxel environment, and secondly to represent BBAs corresponding only to non ambiguous knowledge over singletons. It results that the $\{l u\}$ and $\{h u\}$ centroids are updated iteratively in the FCM algorithm using only certain data. It allows to avoid bias induced by noise and PVE. Before the disjunctive combination, a discounting process (Eq. 1] $X=\varnothing$ ) is performed as follows. Let $V_{i}$ be the considered voxel and $V_{k}$ one of its neighbors. Because of the distance separating them, $V_{k}$ provide less reliable information than $V_{i}$. We propose to discount each neighbor $V_{k}$ according to a coefficient $\alpha_{k}=\exp \left(-\left(V_{k}-V_{i}\right)^{2} / \sigma^{2}\right)$ depending on the distance between $V_{i}$ and $V_{k}$, with $\mathrm{FWHM}^{2}=8 \log 2 . \sigma^{2}$ the Full Width at Half Maximum $(=6 \mathrm{~mm})$ corresponding to the spatial resolution of the image.

The second step focuses on the reduction of imperfect data by combining neighboring voxels using Dempster's rule (Eq. 4). It tends to reduce the ambiguity brought by noise. Before doing this combination, the neighboring voxels are discounted in a same way as previously mentioned, with $X=\Omega$ (Eq. 1).

Segmentation. A labeling process is finally carried out by assigning, to each voxel, the $\{l u\}$ or $\{h u\}$ class having the highest belief mass.

\subsection{Parametric Images for Dose Painting}

Frame of Discernment and Information Sources. Now considering dose painting application from multi-tracer PET images, five classes are distinguished, namely $N$ ormal tissue $\{N\}$, those with an important glucose $M$ etabolism $\{M\}$, an important cell Proliferation $\{P\}$, a significant $H$ ypoxia $\{H\}$, and tissues with a $F$ ull uptake $\{F\}$ : where tissues need an increasing of both the radiation therapy frequency and the dose. The frame of discernment for the three PET images is now: $\Omega=\{N, M, P, H, F\}$. For each voxel of the three modalities, BBAs are assigned to 32 hypotheses: $2^{\Omega}=\{\varnothing,\{N\},\{M\},\{P\}, \ldots,\{N, M\}, \ldots, \Omega\}$.

BBA Estimation. Considering separately each of the three PET images, BBA estimation as proposed in the previous section is first applied in order to assign to each voxel BBAs over $\{l u\},\{h u\}$ and $\{l u, h u\}$. For dose painting, a new frame of discernment given in Table 1 is proposed according to medical interpretation of PET images. It assumes that a low uptake of FDG corresponds to a normal tissue, that a high uptake of FDG can possibly correspond to a high or low uptake of FLT or FMiso, and that a low uptake of FLT and FMiso leads not necessarily to the presence of normal tissue. 
Table 1. Hypotheses considered after BBA estimation for multi-tracer PET images

\begin{tabular}{c|ccc}
\hline Image & $\{\boldsymbol{l u}\}$ & $\{\boldsymbol{h u}\}$ & $\{\boldsymbol{l u}, \boldsymbol{h u}\}$ \\
\hline FDG & $\{N\}$ & $\{M, P, H, F\}$ & $\{N, M, P, H, F\}$ \\
FLT & $\{N, M, H\}$ & $\{P, F\}$ & $\{N, M, P, H, F\}$ \\
FMiso & $\{N, M, P\}$ & $\{H, F\}$ & $\{N, M, P, H, F\}$
\end{tabular}

\section{Fusion of Multi-tracer PET Images for Parametric Image Construction.} First, a registration of the three CT scans is performed using mutual information [1]. Then, the obtained registration parameters are applied to PET images. After BBA estimation, we propose to fuse each voxel of the three PET images using conjunctive combination rule (Eq. 33). First, it allows to obtain the distinction between $\{M\},\{P\},\{H\}$ and $\{F\}$ hypotheses which are under consideration regarding dose painting. The conflict corresponding to a high uptake of FLT or FMiso and a low uptake of FDG is transfered on the empty set, allowing to highlight areas of conflict between the different PET images.

Segmentation. A labeling process is carried out in the same way as presented in the previous section, but considering $\{N\},\{M\},\{P\},\{H\}$ and $\{F\}$ hypotheses.

\section{$3 \quad$ Experiments and Results}

The segmentation method was validated on PET images of an anthropomorphic torso phantom (Data Spectrum Corporation ${ }^{T M}$, Hillsoborough, NC, USA) filled with organ inserts (liver, lungs and spine) and 15 spheres whose volumes vary from 1 to $98 \mathrm{~mL}$. The contrast varies from 4.7 to 10.1 (background), 1.9 to 4.1 (liver) and 30.2 to 65.2 (lung). Our method was compared with a classical FCM 10] and with the Fuzzy Locally Adaptive Bayesian method (FLAB [4]). The relative error rate is used as measure criterion, given by: $E=\left|100 \cdot \frac{V_{s}-V_{a}}{V_{a}}\right|(\%)$, where $V_{s}$ and $V_{a}$ are respectively the measured volume using one of the methods and the actual volume of the sphere. Due to the large amount of spheres and contrasts, mean and standard deviation are computed according to the contrasts and several ranges of sphere volume. The results are given in Fig. 1. It can be seen that our method gives, most of the time, better results than FCM, especially for the smallest spheres. This is due to the centroid update using only nonambiguous data. Moreover, our method outperforms FLAB for spheres whose volume is larger than $3 \mathrm{~mL}$.

Our segmentation method and creation of parametric images for dose painting were carried out on 5 patients who underwent a curative radiotherapy for paramediastinal non-small-cell lung cancer with no movement. The acquisition of PET images (FDG, FLT, and FMiso) were performed on a Biograph Sensation 16 Hi-Rez PET/CT device (SIEMENS Medical Solution, Knoxville, TN, USA) in a time interval less than 72 hours between each exam. Images were corrected 


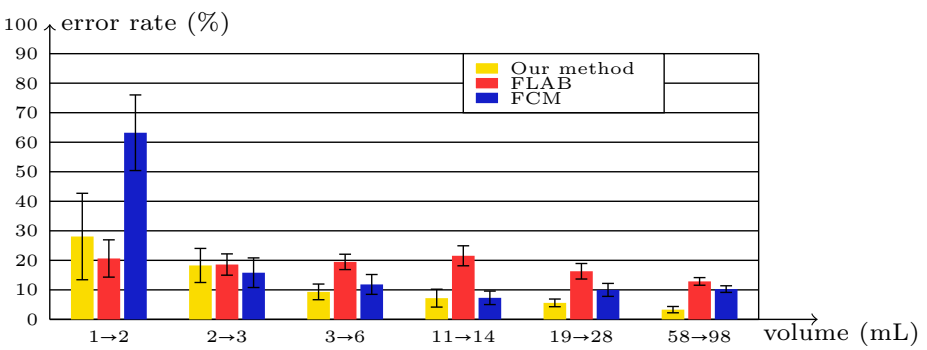

Fig. 1. Comparison of mean error rates according to different sphere volumes using our segmentation method, FLAB [4] and FCM [10]

for dead time, random, scatter and attenuation. The reconstruction was done using an attenuation weighted ordered subset expectation maximization algorithm (4 iterations, 8 subsets). A gaussian post filtering was applied (FWHM $=5 \mathrm{~mm}$ ).

An example of results is given for one patient. Fig. 2 gives an illustration of the different steps of the segmentation process on FDG-PET image. Fig. 2(a) presents the primitive tumor localized in the Region Of Interest (ROI). The results obtained at the different steps of the algorithm are given in Fig. 2(b) to (h), as well as the final segmentation result. Using the first BBA estimation step, the belief masses are spread over the hypotheses $\{l u\},\{h u\}$ and $\{l u, h u\}$ (Fig. 2(b), (c) and (d) respectively). The belief of each voxel for which the information is ambiguous in its neighborhood (noise, PVE) is mainly represented on the hypothesis $\{l u, h u\}$ (cf. Fig. 2(d) ). The result of the segmentation after the second BBA estimation is given in Fig. 2(e), (f) and (g), This step provides high beliefs in favor of $\{l u\}$ and $\{h u\}$ because the uncertainty due to noise has been reduced by neighboring information fusion. The belief at the transitions between the two classes is mainly represented on the hypothesis $\{l u, h u\}$, where imprecision due to PVE is present. Finally, in order to segment $\{h u\}$, voxel labeling is done. The segmented tumor is given in Fig. 2(h).

Results on multi-tracer PET images of the same patient (FDG, FLT, and FMiso) are given Fig. 3(a) (b) and (c) respectively. First applying our BBA estimation method on each image, and then merging these multiple BBAs, we obtain the results as presented Fig. 3. It shows both the BBA corresponding

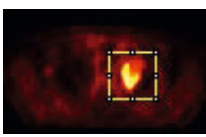

(a) FDG

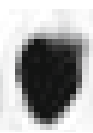

(b) $\{l u\}$

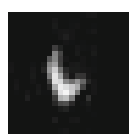

(c) $\{h u\}$

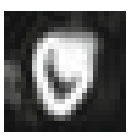

(d) $l u, h u$

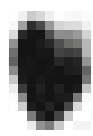

(e) $\{l u\}$

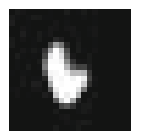

(f) $\{h u\}$

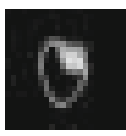

(g) $l u, h u$

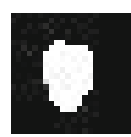

(h)

Fig. 2. Illustration of the segmentation method on PET-FDG image. (a) is the initial image. (b) (c) and (d) show BBAs using the first step of the method in the ROI. (e) (f) and (g) show the BBAs using the second step. (h) presents segmentation result. 


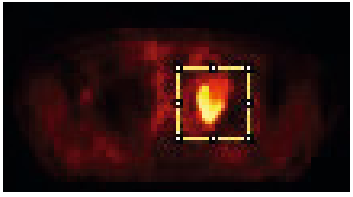

(a) FDG

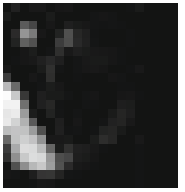

(d) $\varnothing$

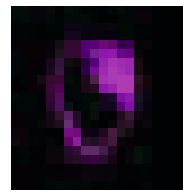

(e) $\{M\}$

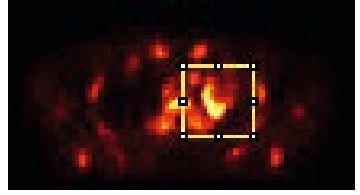

(b) FLT

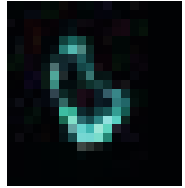

(f) $\{P\}$

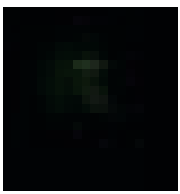

(g) $\{H\}$

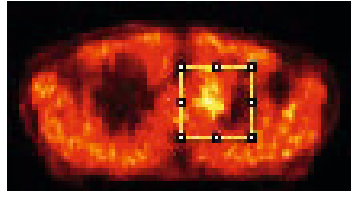

(c) FMiso

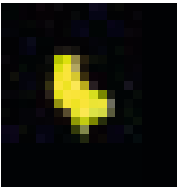

(h) $\{F\}$

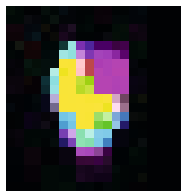

(i)

Fig. 3. Images showing results of the method of information fusion on multi-tracer PET images. (a) (b) and (c) are the initial images using FDG, FLT and FMiso tracers. (d) (e) (f) (g) and (h) are the parametric images applying first the BBA estimation of each ROI of each image and then fusing these BBAs. The final result presenting how to achieve dose painting is presented in (i) and corresponds to the segmented regions.

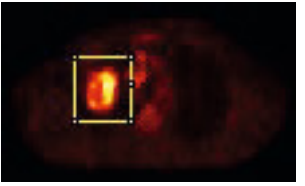

(a) FDG

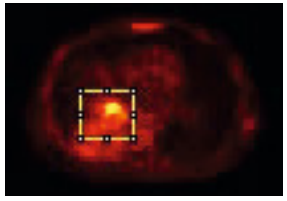

(e) FDG

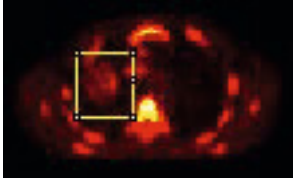

(b) FLT

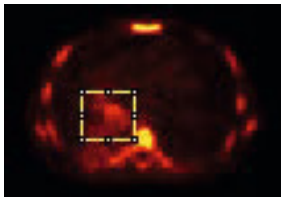

(f) FLT

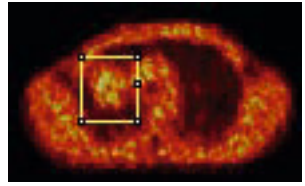

(c) FMiso

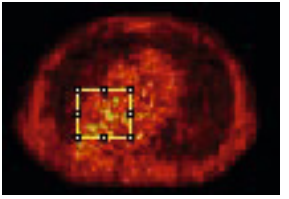

(g) FMiso

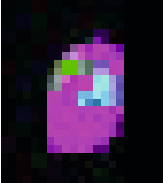

(d)

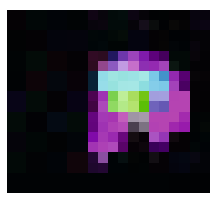

(h)

Fig. 4. Results of our information fusion method on multi-tracer PET images for two patients. (d) and (h) are the fusion results of (a) (b), (c) and (e), (f), (g) respectively.

to the conflict (Fig. 3(d) , and the BBAs corresponding to the hypotheses of interest: $\{M\},\{P\},\{H\}$ and $\{F\}$ (Fig. 3(e) to (h) respectively). Finally, Fig. 3(i) shows the parametric image which provides the distribution information about the three phenomena (glucose metabolism, cellular proliferation and hypoxia) over the pathological region.

The results using our fusion method for two other patients presenting high uptake of FDG, FLT and FMiso are given in Fig. 4. The parametric images given in Fig. 4(d) and (h) and also 3(i) can help radiation oncologist to decide if a classical treatment is needed or dose painting. As a clinical point of view, he can consider three radiotracers independently to delineate BTVs, or our parametric 
images in order to achieve a dose escalation on hypoxic region (green $(H)$ and yellow $(F)$ regions) as well as an increase of dose delivery frequency on high proliferative cells (blue $(P)$ and yellow $(F)$ regions).

\section{Conclusion}

A framework has been developed for segmentation of BTVs and creation of 3D parametric images for dose painting using BFT. An information fusion between neighboring voxels is achieved for segmentation. An information fusion is also done using multi-tracer PET images (FDG, FLT and FMiso) for dose painting. Evaluation of BTV segmentation on an anthropomorphic phantom shows the good performances of the method if compared to two other approaches based on fuzzy sets and probability theory. Even if these first results on 5 patient data are promising for dose painting, they have to be confirmed on a larger database. Nevertheless, the ability of belief function theory to merge information opens very interesting prospects in medical image processing.

\section{References}

1. Ling, C.C., Humm, J., Larson, S., Amols, H., Fuks, Z., Leibel, S., Koutcher, J.A.: Towards multidimensional radiotherapy (MD-CRT): biological imaging and biological conformality. Int. J. Radiat. Oncol. Biol. Phys. 47, 551-560 (2000)

2. Vauclin, S., Doyeux, K., Hapdey, S., Edet-Sanson, A., Vera, P., Gardin, I.: Development of a generic thresholding algorithm for the delineation of ${ }^{18}$ FDG-PETpositive tissue: application to the comparison of three thresholding models. Phys. Med. Biol. 54(22), 6901-6916 (2009)

3. Geets, X., Lee, J.A., Bol, A., Lonneux, M.: A gradient-based method for segmenting FDG-PET images: methodologie and validation. Eur. J. Nucl. Med. Mol. Imaging 34, 1427-1438 (2007)

4. Hatt, M., Cheze-Le Rest, C., Turzo, A., Roux, C., Visvikis, D.: A Fuzzy Locally Adaptive Bayesian Segmentation Approach for Volume Determination in PET. IEEE Trans. Med. Imaging 28(6), 881-893 (2009)

5. Belhassen, S., Zaidi, H.: A novel fuzzy C-means algorithm for unsupervised heterogeneous tumor quantification in PET. Med. Phys. 37, 1309-1324 (2010)

6. Dempster, A.P.: Upper and lower probabilities induced by a multivalued mapping. Ann. Math. Stat. 38, 225-339 (1967)

7. Shafer, G.: A mathematical theory of evidence. Princeton University Press, Princeton (1976)

8. Smets, P., Kennes, R.: The Transferable Belief Model. Artif. Intell. 66, 191-234 (1994)

9. Bloch, I.: Defining belief functions using mathematical morphology - Application to image fusion under imprecision. Int. J. of Approx. Reason. 48(2), 437-465 (2008)

10. Bezdek, J.C.: Pattern Recognition with Fuzzy Objective Function Algorithms. Plenum Press, New York (1981)

11. Viola, P., Wells III, W.M.: Alignment by Maximization of Mutual Information. Int. J. Comput. Vision 24(2), 137-154 (1997) 